\title{
The effect of motoric fluency on metamemory
}

\author{
Jonathan A. Susser • Neil W. Mulligan
}

Published online: 21 November 2014

(C) Psychonomic Society, Inc. 2014

\begin{abstract}
Prior research has demonstrated that certain types of fluency can influence memory predictions, with more fluent processing being associated with greater memory confidence. However, no study has systematically examined whether this pattern extends to the fluency of motoric output. The current study investigated the effect of a motoric-fluency manipulation of hand dominance on judgments of learning (JOLs) and memory performance. Participants predicted better memory for fluently written than nonfluently written stimuli despite no differences in actual recall. A questionnairebased study suggested that the effect of motoric fluency on predictions was not due to peoples' a priori beliefs about memory. These findings are consistent with other fluency effects on JOLs.
\end{abstract}

Keywords Metamemory $\cdot$ Motoric fluency $\cdot$ Judgments of learning $\cdot$ Memory $\cdot$ Recall

Memory monitoring is a central component of metamemory and often is studied by eliciting predictions about future memory performance. Monitoring is important because it influences how people allocate cognitive resources and control later study. Researchers have documented a variety of cues and heuristics that people use to make memory predictions (Bjork, Dunlosky, \& Kornell, 2013). Specifically, processing fluency (Alter \& Oppenheimer, 2009), such as ease of perception (e.g., Besken \& Mulligan, 2014) or ease of encoding (e.g., as measured by self-paced study time, Koriat \& Ma'ayan, 2005), has been shown to influence these

J. A. Susser $(\bowtie) \cdot N$. W. Mulligan $(\bowtie)$

Department of Psychology, University of North Carolina at Chapel

Hill, Davie Hall 346, CB\#3270, Chapel Hill, NC 27599-3270, USA

e-mail: josusser@unc.edu

e-mail: nmulligan@unc.edu judgments. However, fluency is not necessarily associated with better memory performance, and therefore the use of fluency cues can produce metacognitive illusions (e.g., Benjamin, Bjork, \& Schwartz, 1998; Besken \& Mulligan, 2013).

One possible role for fluency in metamemory is captured by the perceptual fluency hypothesis, which proposes that material easily perceived is judged to be more memorable than material less-easily perceived (Rhodes \& Castel, 2008; cf. Mueller, Dunlosky, Tauber, \& Rhodes, 2014). For example, Besken and Mulligan (2014) found a crossed double dissociation between memory predictions and actual memory performance using degraded auditory stimuli. Participants heard digitized recordings of study words that were either intact or interspersed with very brief silences, making a judgment of learning (JOL) for each one in which they rated their confidence in remembering the word on a later test. In accordance with the perceptual fluency hypothesis, participants provided higher JOLs for intact items; however, recall performance was actually greater for the more-difficult-to-perceive degraded items (see also, Besken \& Mulligan, 2013).

In a similar manner, the ease of identifying or generating to-be-remembered information can influence JOLs. Benjamin et al. (1998) had participants generate answers to trivia questions and judge the likelihood that these answers would be remembered on a later recall test. Answers that were generated quickly were given higher JOLs even though the less fluentlygenerated answers actually produced better memory. That is, the ease with which the to-be-remembered information (the answer) was identified was mistakenly taken as an indicator of the item's memorability on a later episodic memory test (see Matvey, Dunlosky, \& Guttentag, 2001, for a similar result regarding generation fluency).

Consistent with the effects of perceptual, encoding, and retrieval fluency, the present experiments assess whether the fluency of motoric output likewise influences metamemory judgments. Alban and Kelley (2013) showed that perceptions 
of physical weight influence JOLs, but a type of motoric fluency has not been systematically examined in metamemory. ${ }^{1}$ Importantly, this fluency does impact other judgments (e.g., Hayes, Paul, Beuger, \& Tipper, 2008). For example, Briñol and Petty (2003) had participants write down three traits about themselves with either their dominant or nondominant hand (the manipulation of motoric fluency) and rate their confidence in those traits. Results showed that participants were more confident in traits written with their dominant hand.

The current study investigates the role of motoric fluency in memory monitoring. Using a hand-dominance manipulation, our main goal was to examine whether this form of fluency influences predictions, consistent with manipulations of perceptual and encoding fluency. To foreshadow, we found an effect of motoric fluency on JOLs in two experiments, and therefore, a second goal of the study was to try to understand how this cue might inform predictions. Specifically, a third experiment assessed whether beliefs, rather than fluency per se, might be contributing to the JOL effect (see Koriat, Bjork, Sheffer, \& Bar, 2004; Mueller et al., 2014).

\section{Experiment 1}

Experiment 1 was an initial assessment of whether motoric fluency influences JOLs. Participants copied down study words with either their dominant or nondominant hand, and aggregate JOLs were taken at the end of the study list. The primary focus is on JOLs but recall deserves brief comment. There is no basis for predicting an effect of hand on recall performance. While some forms of encoding difficulty can enhance memory, such as the perceptual-interference and generation manipulations (e.g., Mulligan \& Lozito, 2004), these manipulations typically affect the initial perception or comprehension of a stimulus. There is no reason to predict an effect on recall, in either direction, with a manipulation of motoric fluency.

\section{Method}

Participants Sixteen undergraduates participated in exchange for course credit.

Materials and design The critical study words were 40 fourletter words, half of which were high frequency (100-500 per million) and half low frequency (1-22; Kucera \& Francis,

\footnotetext{
${ }^{1}$ Some studies of encoding or generating fluency have included a motor response but in these studies the fluency of the motoric response was not experimentally manipulated nor was it the focus of the inquiry (e.g., Benjamin et al., 1998; Hertzog, Dunlosky, Robinson, \& Kidder, 2003; Matvey et al., 2001).
}

1967). ${ }^{2}$ Four additional words were presented at the beginning and end of the list as primacy and recency buffers, and two more were used as practice. Writing hand and word frequency were factorially manipulated within subjects. Words were pseudo-randomly intermixed such that no more than two items of a hand condition were presented consecutively. Two versions of the list were constructed, counterbalancing words across hand.

Procedure During the study phase, participants viewed individual words on a computer screen in preparation for a later memory test. The words Right Hand or Left Hand were displayed below the word on the right or left side of the screen, respectively, to indicate the hand condition. Participants were instructed to print each word with the appropriate hand on a note card, as quickly and accurately as possible. After the word was written, participants pressed the Space Bar, recording the time required to write the word. The word remained on the screen for $13 \mathrm{~s}$.

Next, participants completed a 3-minute distractor task consisting of arithmetic problems. Then participants made aggregate JOLs, being asked to predict the percentage (from 0 to 100) of words written with a given hand that they would recall on the upcoming test. The question was asked separately for right-hand and left-hand words, and the order of the predictions was counterbalanced across participants. After the predictions, participants completed a 5-minute recall test and were then given the Edinburgh Handedness Inventory (Oldfield, 1971) to assess handedness. Right-hand and lefthand items and JOLs were coded as dominant or nondominant hand depending on the handedness of the participant.

\section{Results and discussion}

Fourteen of the 16 participants were right handed. To examine writing times during the study phase, a 2 (hand: dominant or nondominant) $\times 2$ (word frequency: high or low) repeatedmeasures ANOVA was conducted on median writing times (Table 1). ${ }^{3}$ This analysis revealed only a significant effect of hand, $F(1,15)=120.66, p<.001, M S E=1.20 \times 10^{6}, \eta_{\mathrm{p}}^{2}=$ 0.89 , indicating that writing times were longer for the nondominant than dominant hand (the other effects were nonsignificant, $p \mathrm{~s}>0.15)$.

\footnotetext{
${ }^{2}$ High- and low-frequency words were included in anticipation of Experiment 2, in which item-by-item JOLs were collected. Word frequency has sometimes, but not always, affected JOLs (e.g., Benjamin, 2003; cf. Tullis \& Benjamin, 2012). We deemed it possible that if frequency influenced JOLs, this factor might interact with motoric fluency. We return to this issue in Experiment 2.

${ }^{3}$ Writing times were excluded on less than $1 \%$ of the trials because participants either failed to press the Space Bar after writing a word or did not complete the word within the time given.
} 
Table 1 Mean median writing times (and SE) in seconds for experiments 1 and 2

\begin{tabular}{lllll}
\hline & \multicolumn{2}{l}{ Experiment 1} & & \multicolumn{2}{l}{ Experiment 2 } \\
\cline { 2 - 4 } & High frequency & Low frequency & High frequency & Low frequency \\
\hline Dominant & $4.02(0.24)$ & $4.25(0.28)$ & $4.99(0.32)$ & $5.30(0.34)$ \\
Nondominant & $7.12(0.40)$ & $7.16(0.37)$ & $8.60(0.44)$ & $8.41(0.44)$ \\
\hline
\end{tabular}

Analysis of aggregate JOLs showed that participants predicted better memory for dominant $(M=33.13 \%, S E=4.74)$ than nondominant $(M=26.25 \%, S E=4.60)$ items, $t(15)=$ $2.74, p=.015, d=0.69$.

Lastly, a 2 (hand) $\times 2$ (word frequency) ANOVA conducted on participants' recall scores (Table 2 ) revealed no main effect of hand, $F<1$, or word frequency, $F(1,15)=1.90, p=.189$, $M S E=0.01, \eta_{\mathrm{p}}^{2}=0.11$, but a significant interaction between the two, $F(1,15)=6.91, p=.019, M S E=0.01, \eta_{\mathrm{p}}^{2}=0.32$. Follow-up $t$ tests indicated that for high-frequency items, there was a trend for participants to remember more words written with their nondominant than dominant hand, $t(15)=1.86, p=$ $.083, d=0.47$. No significant difference in recall performance was found for low-frequency words, but the trend was in the opposite direction, $t(15)=1.66, p=.118, d=0.43$. Given that we made no prediction about an effect of hand dominance on recall (nor predicted an interaction with word frequency), we defer interpreting this result until after we evaluate its replicability in Experiment 2.tgroup

The writing times verify that the hand manipulation affected motoric fluency: participants took longer to copy down words with their nondominant than dominant hand. More critically, motoric fluency was used as a cue for metamemory judgments, with participants assigning higher JOLs for dominant than nondominant items. This pattern is consistent with other metamemory research showing that JOLs are sensitive to fluency manipulations (e.g., Benjamin et al., 1998; Susser, Mulligan, \& Besken, 2013) and research more specifically on the impact of motoric fluency on other, non-metamemorial judgments (e.g., Briñol \& Petty, 2003).

\section{Experiment 2}

The effect of hand dominance on aggregate JOLs is consistent with the idea that motoric fluency influences memory predictions. However, to assess online monitoring processes, researchers frequently use item-by-item JOLs. In order to replicate the prior result and extend it to this second JOL measure, Experiment 2 used item-by-item predictions.

Additionally, research on metamemory emphasizes the contribution of both experience-based and belief-based information to monitoring. Experience-based processes reflect the subjective experience of interacting with materials. Belief- or theory-based processes, however, are defined as heuristics or beliefs about how memory operates independent of specific online experiences (e.g., Koriat et al., 2004). Hypotheses about fluency and metamemory (like the possibility of a motoric fluency effect) generally focus on experience-based influences. However, it is not yet clear whether the effect of hand dominance in Experiment 1 actually stemmed from experienced fluency per se or reflected a belief-based theory. It has been argued that aggregate JOLs are influenced by both experience and beliefs, whereas item-by-item JOLs are predominantly guided by experience-based information (Koriat et al., 2004; cf., Mueller et al., 2014). Consequently, if the hand-dominance effect is due to experienced motoric fluency, we expect it to persist using item-by-item JOLs.

Method

Participants Seventeen undergraduates participated in exchange for course credit.

Materials, design, and procedure The methods were identical to Experiment 1 except for the use of item-by-item instead of aggregate JOLs. After the $13 \mathrm{~s}$ presentation of each word, participants rated their confidence in remembering the word on the later test [from 0 (not at all confident) to 100 (extremely confident)].

\section{Results and discussion}

One participant took over three standard deviations longer to write down the words than the average participant and was replaced, leaving a sample of 16 (15 right-handed).

A 2 (hand) $\times 2$ (word frequency) ANOVA conducted on participants' median writing times (Table 1) revealed a significant effect of hand, $F(1,15)=130.72, p<.001, M S E=1.38 \times$ $10^{6}, \eta_{\mathrm{p}}^{2}=0.90$, indicating longer writing times for the nondominant than dominant hand, no effect of frequency, $F<1$, and a marginally significant interaction, $F(1,15)=3.45, p=$ $.083, M S E=3.00 \times 10^{5}, \eta_{\mathrm{p}}^{2}=0.19$, indicating a trend for high-frequency words to be written faster than low-frequency words with the dominant hand, but not with the nondominant hand. ${ }^{4}$

\footnotetext{
${ }^{4}$ Writing times were excluded on $2.1 \%$ of the trials for the same reason as in the prior experiment.
} 
Table 2 Mean recall performance (and SE) for experiments 1 and 2

\begin{tabular}{lllll}
\hline & \multicolumn{2}{l}{ Experiment 1} & & \multicolumn{2}{l}{ Experiment 2 } \\
\cline { 2 - 4 } & High frequency & Low frequency & High frequency & Low frequency \\
\hline Dominant & $0.09(0.03)$ & $0.18(0.03)$ & $0.17(0.03)$ & $0.18(0.04)$ \\
Nondominant & $0.14(0.03)$ & $0.13(0.02)$ & $0.21(0.04)$ & $0.13(0.03)$ \\
\hline
\end{tabular}

A 2 (hand) $\times 2$ (word frequency) ANOVA conducted on participants' JOLs (Fig. 1) revealed only a significant main effect of hand, $F(1,15)=11.28, p=.004, M S E=24.19, \eta_{\mathrm{p}}^{2}=$ 0.43 , indicating higher JOLs for dominant than nondominant items (other effects, $p \mathrm{~s}>.15$ ).

In order to further dissect the relationship between motoric fluency and JOLs, a partial correlation analysis was conducted. Such an analysis assesses the mediating effect of experienced fluency (in this case measured by writing times) on the hand-JOL relationship (see Mueller et al., 2014). Withinsubject Kim's d correlations (Gonzalez \& Nelson, 1996) between hand ( $0=$ nondominant; $1=$ dominant) and JOLs revealed a relationship significantly greater than $0, d=.07$, $S E=0.03, t(15)=2.59, p=.021 .^{5}$ When controlling for writing times, this relationship significantly decreased (partial $d=.02, S E=0.01), t(15)=2.52, p=.024$, and did not differ from $0, t(15)=1.38, p=.187$. Additionally, the gamma correlation (Nelson, 1984) between writing times and JOLs was weak but significantly less than $0, G=-.08, S E=0.02$, $t(15)=3.55, p=.003$. This set of results is consistent with the proposal that the effect of hand dominance on JOLs is at least partly mediated by experienced motoric fluency.

A 2 (hand) $\times 2$ (word frequency) ANOVA conducted on recall performance (Table 2) revealed no significant effects (all $p s>.20$ ). Finally, JOL resolution was relatively low but significantly greater than $0, G=.17, S E=0.08, t(15)=2.19, p$ $=.045$, and did not vary across hand dominance, $t<1$.

The effect of hand dominance on writing times again validates the manipulation of motoric fluency. Also consistent with Experiment 1, participants gave higher predictions for items written with the dominant than nondominant hand. These findings, along with the mediation analysis, provide further support that motoric fluency is a cue for memory predictions and suggest fluency experience is playing a role in this effect.

Word frequency did not have an influence on JOLs, although numerically the pattern was in the expected direction. While some prior research has shown that high-frequency words are judged as more memorable, possibly due to increased ease of processing (Begg, Duft, Lalonde, Melnick, \& Sanvito, 1989; Benjamin, 2003), this result is not always

\footnotetext{
${ }^{5}$ Gonzalez and Nelson (1996) recommend using Kim's $d_{x y}$ when ties on the predictor variable (in this case, hand) are unambiguous.
}

found (Tullis \& Benjamin, 2012). The effect of word frequency on metamemory may be weak and inconsistent, and the addition of the hand manipulation may have interfered with any potential effect. Regarding recall performance, no significant effects were found. These results are largely consistent with Experiment 1 and suggest that motoric disfluency does not influence memory performance (we return to this point below). Finally, the null effect of word frequency on free recall is typical for mixed-list designs (in contrast to pure-list designs in which high-frequency words produce greater recall, e.g., MacLeod \& Kampe, 1996).

\section{Experiment 3}

Experiments 1 and 2 suggest that motoric fluency, as implemented by hand dominance, affects JOLs, and Experiment 2, more specifically, implies that experienced fluency rather than beliefs contributes to the effect. However, to further isolate peoples' mnemonic beliefs about motoric fluency, a questionnaire was designed. This is a standard method for attempting to isolate theory-based influences on metamemory (see Koriat et al., 2004; Mueller et al., 2014). In this design, participants read a description of an experiment and predict recall performance for different conditions without actually having the experience (e.g., of varying degrees of perceptual or motoric fluency) that participants in the actual experiment have.

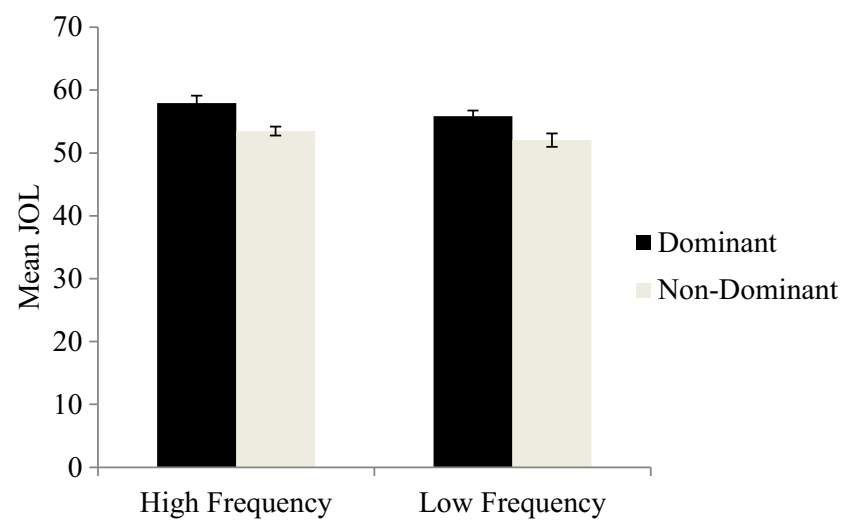

Fig. 1 Mean JOLs in Experiment 2. Error bars represent $\pm 1 \mathrm{SE}$ (within-subject error bars computed using method outlined by Cousineau, 2005) 
Because participants are not presented with any stimuli, there is no influence of direct experience on predictions.

\section{Method}

Participants Sixty undergraduates participated in exchange for course credit.

Materials, design, and procedure Participants read the following description:

In a previous experiment, participants were presented with 48 four-letter words on the computer screen, one at a time. Their task was to try to learn the words for a later memory test. They also were required to copy each word down, in print, on a note card with either their dominant or nondominant hand. Participants were given $13 \mathrm{sec}-$ onds to write and study the word before the next one was presented. In all, half of the words were written with participants' dominant hands, and the other half were written with participants' nondominant hands.

Participants then solved math problems in their heads for 3 minutes. Finally, they were asked to write down as many words as they could remember from the first part of the experiment. They could write the words in any order that they wanted.

Participants in the current experiment then estimated the percentage of words of each type (dominant and nondominant) that students recalled. The order of the predictions was counterbalanced across participants.

\section{Results and discussion}

A paired-samples $t$ test on predictions revealed no difference between dominant $(M=46.80 \%, S E=2.53)$ and nondominant $(M=49.37 \%, S E=2.75)$ items, $t<1$. This result suggests that people do not have a priori beliefs about an effect of hand dominance on memory and therefore indicates that the results of Experiments 1 and 2 were not driven by any such beliefs. Indeed, peoples' beliefs as reflected in this questionnaire are more in line with actual memory performance, given the lack of any reliable effect in recall across Experiments 1 and 2. Therefore, it is possible that the direct experience overrides peoples' beliefs (see also Koriat et al., 2004). An open question is whether people can learn from their experience across multiple cycles, as has been demonstrated with other illusions (e.g., Tauber \& Rhodes, 2010).

Because this is a null result, it is important to consider the power of the analysis. The trend is in the opposite direction of the results of Experiments 1 and 2, making it unlikely that the present results are simply a low-power artifact. However, a power analysis was conducted, based on the average effect size of hand-dominance on JOLs in Experiments 1 and $2(d=$ 0.77). The analysis revealed a power exceeding .99 to detect an effect of this size, and exceeding .97 for an effect size 1/3 smaller. Thus, there was substantial power to detect an effect even considerably smaller than that found in Experiments 1 and 2.

\section{General discussion}

Consistent with research on perceptual fluency, motoric fluency, as indexed by a hand-dominance manipulation, informs memory predictions. Participants gave higher JOLs to words they copied down in a fluent matter (with their dominant hand) than in a disfluent manner (with their nondominant hand). This was found with aggregate and item-by-item JOLs. Furthermore, a questionnaire-based study revealed no evidence that the effect is driven by a priori beliefs about handedness.

It may be worth thinking about our experiments as reflecting a continuum of influences, with Experiment 2 (item-by-item JOLs) tapping experience most strongly, Experiment 1 (aggregate JOLs) assessing experience or online beliefs, and Experiment 3 (questionnaire) gauging beliefs uncontaminated by any experience. The pattern of results across the three experiments - coupled with the mediation analysis in Experiment 2-suggest a JOL effect with at least a partial experiential basis.

One straightforward experience-based account would state that the motoric disfluency produced by the nondominant hand might lend the stimulus a feeling of uncertainty or unfamiliarity that results in lower JOLs. Alternatively, the effect of motoric disfluency might be indirect. Briñol and Petty (2003) proposed that hand writing might influence judgments through the appearance of the product of writing. Writing with the nondominant hand yields text that looks shaky and uncertain, which may reduce confidence (in the present case, memorial confidence) in that information. Stated differently, motor disfluency might produce perceptuallydisfluent feedback that drives the effect-a more complex proposal, but still one driven by experience, in this case the experience of disfluent perceptual feedback. Research could examine this possibility by having participants make the motions of writing without being able to see the outcome (e.g., using a stylus without ink). Future investigations are required to tease apart these alternatives.

The effect of motoric fluency on metamemory appears to be a metacognitive illusion given that no consistent effect of hand was found on recall. Although the recall results of Experiment 1 might seem unclear on this point, it should be noted that on average there was no hint of an effect of hand 
dominance in that experiment, a result displayed even more clearly in Experiment 2. Furthermore, ongoing research in our lab examining motoric fluency and metacognitive control has consistently replicated the effect of motoric fluency on JOLs but has consistently found no effect on recall. Thus, motoric fluency affects metamemory but not actual memory performance - a metacognitive illusion similar to that of Alban and Kelley (2013) who found that physical weight impacted JOLs but not recall.

\section{References}

Alban, M. W., \& Kelley, C. M. (2013). Embodiment meets metamemory: Weight as a cue for metacognitive judgments. Journal of Experimental Psychology: Learning, Memory, and Cognition, 39, 1628-1634. doi:10.1037/a0032420

Alter, A. L., \& Oppenheimer, D. M. (2009). Uniting the tribes of fluency to form a metacognitive nation. Personality and Social Psychology Review, 13, 219-235. doi:10.1177/1088868309341564

Begg, I., Duft, S., Lalonde, P., Melnick, R., \& Sanvito, J. (1989). Memory predictions are based on ease of processing. Journal of Memory and Language, 28, 610-632. doi:10.1016/0749-596X(89)90016-8

Benjamin, A. S. (2003). Predicting and postdicting the effects of word frequency on memory. Memory \& Cognition, 31, 297-305. doi:10. 3758/BF03194388

Benjamin, A. S., Bjork, R. A., \& Schwartz, B. L. (1998). The mismeasure of memory: When retrieval fluency is misleading as a metamnemonic index. Journal of Experimental Psychology: General, 127, 55-68. doi:10.1037/0096-3445.127.1.55

Besken, M., \& Mulligan, N. W. (2013). Easily perceived, easily remembered? Perceptual interference produces a double dissociation between metamemory and memory performance. Memory \& Cognition, 41, 897-903. doi:10.3758/s13421-013-0307-8

Besken, M., \& Mulligan, N. W. (2014). Perceptual fluency, auditory generation, and metamemory: Analyzing the perceptual fluency hypothesis in the auditory modality. Journal of Experimental Psychology: Learning, Memory, and Cognition, 40, 429-440. doi: 10.1037/a0034407

Bjork, R. A., Dunlosky, J., \& Kornell, N. (2013). Self-regulated learning: Beliefs, techniques, and illusions. Annual Review of Psychology, 64, 417-444. doi:10.1146/annurev-psych-113011-143823

Briñol, P., \& Petty, R. E. (2003). Overt head movements and persuasion: A self-validation analysis. Journal of Personality and Social Psychology, 84, 1123-1139. doi:10.1037/0022-3514.84.6.1123

Cousineau, D. (2005). Confidence intervals in within-subject designs: A simpler solution to Loftus and Masson's method. Tutorials in Quantitative Methods for Psychology, 1, 42-45.

Gonzalez, R., \& Nelson, T. O. (1996). Measuring ordinal association in situations that contain tied scores. Psychological Bulletin, 119, 159165. doi:10.1037/0033-2909.119.1.159
Hayes, A. E., Paul, M. A., Beuger, B., \& Tipper, S. P. (2008). Self produced and observed actions influence emotion: The roles of action fluency and eye gaze. Psychological Research, 72, 461472. doi:10.1007/s00426-007-0125-3

Hertzog, C., Dunlosky, J., Robinson, A. E., \& Kidder, D. P. (2003). Encoding fluency is a cue used for judgments about learning. Journal of Experimental Psychology: Learning, Memory, and Cognition, 29, 22-34. doi:10.1037/0278-7393. 29.1.22

Koriat, A., \& Ma'ayan, H. (2005). The effects of encoding fluency and retrieval fluency on judgments of learning. Journal of Memory and Language, 52, 478-492. doi:10.1016/j.jml. 2005.01 .001

Koriat, A., Bjork, R. A., Sheffer, L., \& Bar, S. K. (2004). Predicting one's own forgetting: The role of experience-based and theory-based processes. Journal of Experimental Psychology: General, 133, 643-656. doi:10.1037/0096-3445.133.4.643

Kucera, H., \& Francis, W. N. (1967). Computational analysis of presentday American English. Providence: Brown University Press.

MacLeod, C. M., \& Kampe, K. E. (1996). Word frequency effects on recall, recognition, and word fragment completion tests. Journal of Experimental Psychology: Learning, Memory, and Cognition, 22, 132-142. doi:10.1037/0278-7393.22.1.132

Matvey, G., Dunlosky, J., \& Guttentag, R. (2001). Fluency of retrieval at study affects judgments of learning (JOLs): An analytic or nonanalytic basis for JOLs? Memory \& Cognition, 29, 222-233. doi:10. 3758/BF03194916

Mueller, M. L., Dunlosky, J., Tauber, S. K., \& Rhodes, M. G. (2014). The font-size effect on judgments of learning: Does it exemplify fluency effects or reflect people's beliefs about memory? Journal of Memory and Language, 70, 1-12. doi: 10.1016/j.jml.2013.09.007

Mulligan, N. W., \& Lozito, J. P. (2004). Self-generation and memory. In B. H. Ross (Ed.), Psychology of learning and motivation (pp. 175214). San Diego: Elsevier Academic Press.

Nelson, T. O. (1984). A comparison of current measures of the accuracy of feeling-of-knowing predictions. Psychological Bulletin, 95, 109133. doi:10.1037/0033-2909.95.1.109

Oldfield, R. C. (1971). The assessment and analysis of handedness: The Edinburgh inventory. Neuropsychologia, 9, 97-113. doi:10.1016/ 0028-3932(71)90067-4

Rhodes, M. G., \& Castel, A. D. (2008). Memory predictions are influenced by perceptual information: Evidence for metacognitive illusions. Journal of Experimental Psychology: General, 137, 615-625. doi:10.1037/a0013684

Susser, J. A., Mulligan, N. W., \& Besken, M. (2013). The effects of perceptual fluency and list composition on judgments of learning (JOLs). Memory \& Cognition, 41, 1000-1011. doi:10.3758/s13421013-0323-8

Tauber, S. K., \& Rhodes, M. G. (2010). Metacognitive errors contribute to the difficulty in remembering proper names. Memory, 18, 522532. doi:10.1080/09658211.2010.481818

Tullis, J. G., \& Benjamin, A. S. (2012). The effectiveness of updating metacognitive knowledge in the elderly: Evidence from metamnemonic judgments of word frequency. Psychology and Aging, 27, 683-690. doi:10.1037/a0025838 